\title{
Kinematics of the local universe. III. Neutral hydrogen observations of southern galaxies
}

\author{
H. Di Nella ${ }^{1,2}$, G. Paturel ${ }^{1}$, A.J. Walsh ${ }^{2}$, L. Bottinelli ${ }^{3,4}$, L. Gouguenheim ${ }^{3,4}$ and G. Theureau ${ }^{3}$ \\ 1 CRAL-Observatoire de Lyon, F-69561 Saint-Genis Laval Cedex, France \\ 2 Depart. astrophysics, Uni. New South Wales, 2052 Sydney, NSW, Australia \\ 3 Observatoire de Paris-Meudon, ARPEGES, URA CNRS 1757, F-92195 Meudon Principal Cedex, France \\ 4 Université Paris-Sud, F-91405 Orsay, France
}

Received January 11; accepted January 15, 1996

\begin{abstract}
This paper is the third one in a series dedicated to the study of the kinematics of the local universe. It gives 52 new 21-cm neutral hydrogen measurements, made with the $64-\mathrm{m}$ Parkes radio telescope. These new measurements are used to built a complete sample of 5,219 galaxies with $21-\mathrm{cm}$ line widths, radial velocity and apparent diameter. This sample is complete down to a diameter limit of 1.6 arcmin.
\end{abstract}

Key words: galaxies: redshifts; ISM — radio lines: galaxies — catalogue

\section{Introduction}

The main purpose of this series of papers is the study of the velocity field from unbiased distances for a galaxy sample complete down to a given apparent diameter limit of $1.6 \operatorname{arcmin}$ (i.e. $\log D_{25}=1.20$, where $D_{25}$ is the apparent diameter at the brightness level of 25 Bmag. $\operatorname{arcsec}^{-2}$. This diameter is expressed in $0.1^{\prime}$ following the convention adopted by de Vaucouleurs et al. 1976). This program has been presented previously (Paturel et al. 1990). First, it requires radial velocity measurements and then, $21-\mathrm{cm}$ line width measurements, the later ones being used for distance determination through the Tully-Fisher relation (Tully \& Fisher 1977). Radial velocity measurements were thus undertaken at ESO, OHP and Nançay and 21-cm line width measurements at Nançay. Because of the pointing limitation of the Nançay radio telescope ${ }^{1}$, all galaxies in the extreme south (i.e. below $-38 \mathrm{deg}$ ) have to be observed at Parkes. In two previous papers (Bottinelli et al. 1992; Bottinelli et al. 1993), 664 radial velocity measurements were published. In the present paper 52 new HI measurements obtained with the Parkes radio-telescope are given.

\section{Observations}

The observations were conducted in 1994, from August 16 to 20 with the Parkes 64-m radio telescope at Parkes Ob-

Send offprint requests to: H. Di Nella

${ }^{1}$ The transit meridian Nançay radiotelescope can observe for declinations in the range $\approx 90 \mathrm{deg}$ to $-38 \mathrm{deg}$. servatory, ATNF. The sensitivity is $0.63 \mathrm{KJy}^{-1}$ and the receiver system temperature is $50 \mathrm{~K}$. The $\mathrm{HPBW}$ of the telescope is $15^{\prime}$. The 1024-channel autocorrelation spectrometer (Ables et al. 1975) was normally used in the 2 -channel mode, with each receiver sampled in 512 delay channels. Galaxies were observed with $32 \mathrm{MHz}$ bandwith, which gives an effective velocity resolution of $7 \mathrm{~km} / \mathrm{s}$. Integrations of 5 minutes were made on source, followed by similar reference observation made with a declination offset of $+0.5 \mathrm{deg}$. Flux scales for the HI spectra were determined in each polarization channel from observations of the continuum-radio source Hydra A (Davies et al. 1989). The flux density and spectral index $\left(S_{\nu} \sim \nu^{-\alpha}\right)$ of this source were taken to be $S_{\nu}=43.5$ Jy and $\alpha=0.90$ at 1410 MHz. The linear polarization of Hydra A is negligibly small $(<0.3$ per cent).

\section{Results}

Spectra were processed with the Spectral Line Analysis Package (SLAP) given by Staveley-Smith (1985). Some individual bad scans were rejected after examination by eye. Then, the remaining scans were added while spectra of both polarizations are averaged. A baseline is fitted with a polynomial for reconstructing the corrected $21-\mathrm{cm}$ line profile. The adopted order is decided interactively but it is generally about 3 . The $21-\mathrm{cm}$ line profiles are given in the last pages. Each frame is identified with the PGC name according to Table 1. 
The width is measured at two levels, $50 \%$ and $20 \%$ of the peak, leading to uncorrected values $W_{50}$ and $W_{20}$. The systemic velocity is measured as the middle of the $20 \%$ level. It is expressed with the optical convention $c . \Delta \lambda / \lambda$. The integral flux of the calibrated profile gives the observed HI flux, $F_{\mathrm{HI}}$. The signal-to-noise ratio $(S / N)$ is defined as: $F_{\mathrm{HI}} /\left(W_{20} \sigma_{\mathrm{f}}\right)$ (Roth et al. 1994), where $\sigma_{\mathrm{f}}$ is the rms noise in Jy.

We calculated the actual internal errors on the measured heliocentric velocity $(V)$, width at $20 \%(W 20)$ and at 50\% (W50), according to Fouqué et al. (1990):

$\sigma(V)=3.3 \times 1.11 R^{0.5} P^{0.5} S^{-1}$

$\sigma(W 50)=2 \sigma(V)$

$\sigma(W 20)=3 \sigma(V)$

Where $S$ is the signal to noise ratio, $R$ the spectral resolution and $P=(W 20-W 50) / 2$.

From the equation:

$\sigma^{2}=\sigma(V \text { literature })^{2}+\sigma(V \text { radio })^{2}$

A comparison between literature and radio velocities shows that the slope doesn't differ significantly from one and the zero point doesn't differ significantly from zero. We obtain :

$\sigma^{2}=87 \mathrm{~km} \mathrm{~s}^{-1}$

and:

$\sigma(V$ radio $)=7 \mathrm{kms}^{-1}$

thus:

$\sigma(V$ literature $)=87 \mathrm{kms}^{-1}$

This shows that all the error in the comparison comes from the measurements of the literature. This is coherent with the fact that measurements from the literature are essentially optical measurements. This error is also in agreement with the mean error $\left(90 \mathrm{~km} \mathrm{~s}^{-1}\right)$ on optical velocity measurements conducted for the present study (Bottinelli et al. 1992).

The detailed list of observed objects is given in Table 1 . The columns are arranged as follows:

- Column 1: PGC Number according to Paturel et al. (1989)

- Column 2: Name according to a hierarchy (NGC, IC, ESO...)

- Column 3: 1950-RA and DEC. in hr, mn, s and deg.',"

- Column 4: Heliocentric radial velocity (in $\mathrm{km} / \mathrm{s}$ ) from the literature

- Column 5: Heliocentric velocity with its error (optical convention in $\mathrm{km} / \mathrm{s}$ )

- Column 6: Width at $20 \%$ of the peak with its error (optical convention in $\mathrm{km} / \mathrm{s}$ )

- Column 7: Width at 50\% of the peak with its error (optical convention in $\mathrm{km} / \mathrm{s}$ )

- Column 8: HI flux integral (in Jy $\mathrm{km} \mathrm{s}^{-1}$ )

- Column 9: $S / N$ ratio $F_{\mathrm{HI}} /\left(W_{20} \sigma_{\mathrm{f}}\right)$

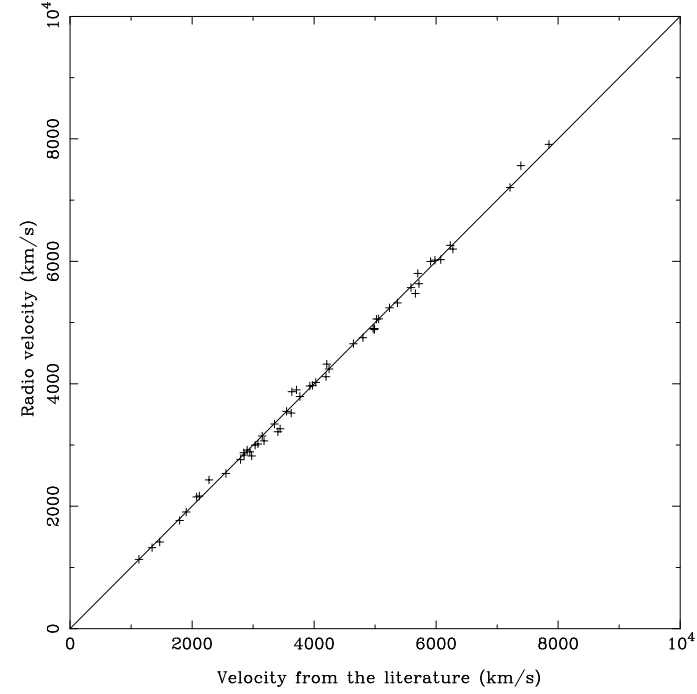

Fig. 1. Comparison between optical and radio velocities. The continuous line is the first bissect. The mean dispersion is: $87 \mathrm{~km} \mathrm{~s}^{-1}$

\section{- Column 10. Note}

\section{Completeness in different regions}

The global completeness of the total sample of 5.219 galaxies was studied in a previous paper (Paturel et al. 1994), but we have not yet tested the completeness in different regions of the sky.

A study is conducted in the hypergalactic plane coordinates $^{2} h g l, h g b$ as defined by Di Nella \& Paturel (1994). This plane contains superclusters of Perseus-Pisces, Pavo-Indus, Centaurus, Coma (and a part of the Great-Wall) and the Local Super Cluster. Two regions are studied (Fig. 2):

- On the side of Centaurus (Great Attractor, see Burstein et al. 1987), i.e. $0 \leq h g l<180$ deg. The corresponding completeness curve is drawn with a dashed line.

- On the opposite side, i.e. $180 \leq h g l<360 \mathrm{deg}$. The corresponding completeness curve is drawn with a dotted line.

The completeness is well fulfilled up to the limit $\log D_{25}=1.2$. This shows that the small deficiency in the total sample, towards the Northern and Southern poles should not affect a study of the kinematics in the so-called hypergalactic plane. However, more HI observations are still needed in the southern hemisphere.

Acknowledgements. We acknowledge L. Staveley-Smith for his support and the staff members at Parkes observatory. We received financial support for this project from the INSU (Institut National des Sciences de l'Univers) under the

${ }^{2}$ hypergalactic plane according to Paturel et al. (1988). 


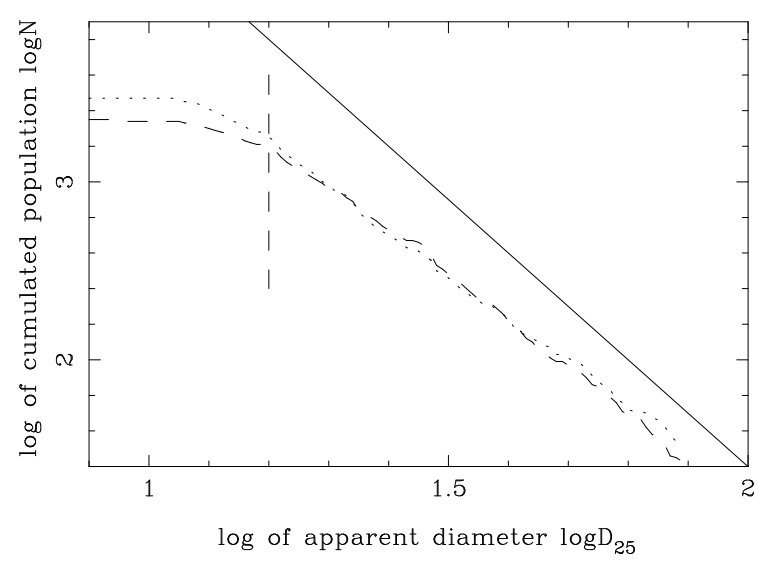

Fig. 2. Completeness curve on the side of the presumed Great-Attractor (dashed line) and on the opposite side (dotted line)

French-Australian Cooperation Scheme and from the 'Conseil Régional Rhone-Alpes' under the program: 'EURODOC'.

\section{References}

Ables J.G., Brooks J.W., Cooper B.F.C., et al., 1975, Rev. Sc. Instr. 46, 284
Bottinelli L., Durand N., Fouqué P., et al., 1992, A\&AS 93, 173

Bottinelli L., Durand N., Fouqué P., et al., 1993, A\&AS 102, 57

Burstein D., Davies R., Dressler A., et al., 1987 in Galaxy Distances and Deviation from the Universal Expansion. In: Madore and Tully (Reidel), p. 123

Davies R.D., Staveley-Smith L., Murray J.D., 1989, MNRAS 236, 171

Di Nella H., Paturel G., 1994, C.R.Acad.Sc. Paris, t.319, p. 57

Fouqué P., Bottinelli L., Durand N., Gouguenheim L., Paturel G., 1990, A\&AS 86, 473

Paturel G., Bottinelli L., Gouguenheim L., Fouqué P., 1988, A\&A 189, 1

Paturel G., Bottinelli L., Fouqué P., et al., 1990, The Messenger 62,8

Paturel G., Bottinelli L., Di Nella H., et al., 1994, A\&A 289, 711

Paturel G., Fouqué P., Bottinelli L., Gouguenheim L., 1989, A\&AS 80, 299 and Extragalactic Database Monographs 1, Vols. 1-3

Roth J., Mould J., Staveley-Smith L., 1994, AJ 108, 851

Staveley-Smith L., 1985, PhD thesis, University of Manchester Tully B., Fisher R., 1977, A\&A 54, 661

Vaucouleurs G. de, Vaucouleurs A. de, Corwin H.G. Jr., 1976, Second Reference Catalogue of Bright Galaxies. University of Texas Press, Austin (RC2) 
TABLE 1. The Table of 52 observed galaxies

\begin{tabular}{|c|c|c|c|c|c|c|c|c|}
\hline $\begin{array}{l}\text { PGC/LEDA } \\
\text { (1) }\end{array}$ & $\begin{array}{l}\text { Name } \\
(2)\end{array}$ & $\begin{array}{l}\text { R.A. } 1950 \text { DEC. } \\
\text { hms }{ }^{\prime} \text {, " }\end{array}$ & $\begin{array}{l}\text { Vlit. } \\
\mathrm{km} / \mathrm{s} \\
(4)\end{array}$ & $\begin{array}{l}\mathrm{V} 20 \\
\mathrm{~km} / \mathrm{s} \\
(5)\end{array}$ & $\begin{array}{l}\text { W20 } \\
\mathrm{km} / \mathrm{s} \\
(6)\end{array}$ & $\begin{array}{l}\text { W50 } \\
\mathrm{km} / \mathrm{s} \\
(7)\end{array}$ & $\begin{array}{l}\mathrm{F}(\mathrm{HI}) \quad \mathrm{S} / \mathrm{N} \\
\mathrm{Jy} \cdot \mathrm{km} / \mathrm{s} \\
(8) \quad(9)\end{array}$ & $\begin{array}{l}\text { Note } \\
\text { (10) }\end{array}$ \\
\hline
\end{tabular}

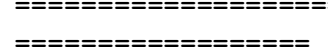

PGC 01532 ESO 242- 7

$002155.0-454706$

PGC 02958 ESO 150- 24 004834.9-555248

PGC 05764 ESO 13- 16 013238.0-794348

PGC 05967 ESO 13- 18 013643.0-803606

PGC 06249 NGC $2573 \quad 024256.1-893412$

PGC 08320 ESO 298- 15 020836.0-410912

PGC 10624 ESO 299- 18 024632.0-404542

PGC 14093 ESO 83- 12 035431.0-660518

PGC 15790 ESO 84- 34 043956.9-631206

PGC 15802 ESO 157- 50 043933.0-525106

PGC 18355 NGC 2187A 060405.9-693524

PGC 18407 ESO 307- 17 060448.9-395124

PGC 20094 ESO 309- 17 070229.0-414818

PGC 20865 ESO 123- 9 072215.0-615548

PGC 21429 ESO 208- 31 073622.0-521124

PGC 21472 ESO 59- 12 073834.0-683918

PGC 21815 ESO 311- 12 074553.0-411936

PGC 26003 ESO 91- 3 091228.0-632512

PGC 26532 ESO 126- 10 092108.0-605006

PGC 28025 ESO 61- 15 094519.0-684100

PGC 29450 ESO $316-29$ 100540.0-410518

PGC 29723 ESO 263- 16

PGC 32660 ESO 264- 48

PGC 35140 ESO 216- 8

PGC 37334 ESO $320-31$

PGC 38841 ESO $321-10$

PGC 47003 ESO 220- 8

PGC 49586 NGC 5365A

PGC 49655 ESO 174- 3

PGC 50330 ESO 175- 1

PGC 50798 ESO 271- 22

PGC 53377 ESO 327- 32

PGC 53527 NGC 5786

PGC 53535 ESO 327- 39

PGC 55256 ESO 329- 7

PGC 58999 ESO 137- 42

PGC 59880 ESO 138- 17

PGC 61532 ESO 71- 4

PGC 61712 ESO 182- 10

PGC 61814 ESO 280- 13

PGC 61850 ESO 335- 11

PGC 61948 ESO 281- 1

PGC 62361 ESO 336- 13

PGC 62411 ESO 336- 16

PGC 62614 ESO 231- 11

PGC 62908 ESO 282- 21 $\begin{array}{lllllllll}5720 & 5635 & 6 & 441 & 18 & 431 & 12 & 2.3 & 3.6\end{array}$

$\begin{array}{lllllllll}7850 & 7911 & 8 & 431 & 24 & 415 & 16 & 5.8 & 3.5\end{array}$

$\begin{array}{lllllllll}1795 & 1767 & 3 & 186 & 9 & 166 & 6 & 11.8 & 10.0\end{array}$

$\begin{array}{lllllllll}4208 & 4322 & 9 & 291 & 25 & 260 & 17 & 6.0 & 4.5\end{array}$

$\begin{array}{lllllllll}2278 & 2429 & 5 & 250 & 16 & 220 & 11 & 9.2 & 6.9\end{array}$

$\begin{array}{llllllllll}1469 & 1415 & 5 & 181 & 14 & 157 & 10 & 10.0 & 7.1\end{array}$

$\begin{array}{lllllllll}4801 & 4752 & 16 & 453 & 48 & 367 & 32 & 14.3 & 4.0\end{array}$

$\begin{array}{lllllllll}5660 & 5474 & 30 & 540 & 89 & 404 & 59 & 6.6 & 2.7\end{array}$

$\begin{array}{lllllllll}6275 & 6199 & 16 & 502 & 49 & 444 & 33 & 8.3 & 3.2\end{array}$

$\begin{array}{lllllllll}3636 & 3871 & 8 & 225 & 25 & 204 & 17 & 7.2 & 3.8\end{array}$

$\begin{array}{lllllllll}3930 & 3964 & 14 & 464 & 43 & 417 & 29 & 3.9 & 3.3\end{array}$

$\begin{array}{lllllllll}4990 & 4902 & 3 & 418 & 10 & 366 & 7 & 12.9 & 14.6\end{array}$

$\begin{array}{lllllllll}5235 & 5240 & 8 & 469 & 24 & 437 & 16 & 8.4 & 4.9\end{array}$

$\begin{array}{lllllllll}3406 & 3216 & 3 & 292 & 8 & 277 & 5 & 15.8 & 10.4\end{array}$

$\begin{array}{lllllllll}3179 & 3068 & 7 & 370 & 22 & 351 & 15 & 7.1 & 4.1\end{array}$

$\begin{array}{lllllllll}1345 & 1323 & 11 & 196 & 33 & 177 & 22 & 2.3 & 2.7\end{array}$

$\begin{array}{lllllllll}1128 & 1131 & 12 & 239 & 36 & 215 & 24 & 2.7 & 2.8\end{array}$

$\begin{array}{lllllllll}1904 & 1906 & 3 & 344 & 9 & 330 & 6 & 14.1 & 9.1\end{array}$

$\begin{array}{lllllllll}2074 & 2152 & 5 & 380 & 16 & 346 & 11 & 7.9 & 7.5\end{array}$

$\begin{array}{lllllllll}4984 & 4885 & 4 & 379 & 12 & 356 & 8 & 25.5 & 8.1\end{array}$

$\begin{array}{lllllllll}5701 & 5803 & 4 & 420 & 13 & 387 & 9 & 5.8 & 9.0\end{array}$

$\begin{array}{lllllllll}4195 & 4116 & 1 & 211 & 3 & 191 & 2 & 16.9 & 28.0\end{array}$

$\begin{array}{lllllllll}2847 & 2874 & 4 & 304 & 11 & 277 & 7 & 15.0 & 10.0\end{array}$

$\begin{array}{llllllllll}5588 & 5570 & 6 & 453 & 18 & 436 & 12 & 11.6 & 4.6\end{array}$

$\begin{array}{lllllllll}2947 & 2889 & 7 & 359 & 20 & 344 & 13 & 8.8 & 4.0\end{array}$

$\begin{array}{lllllllll}3150 & 3147 & 7 & 359 & 21 & 326 & 14 & 4.5 & 5.6\end{array}$

$\begin{array}{lllllllll}2977 & 2821 & 19 & 736 & 56 & 678 & 37 & 10.0 & 2.8\end{array}$

$\begin{array}{llllllllll}2795 & 2760 & 8 & 426 & 24 & 406 & 16 & 4.6 & 3.8\end{array}$

$\begin{array}{lllllllll}4027 & 4023 & 7 & 250 & 21 & 210 & 14 & 26.7 & 6.3\end{array}$

$\begin{array}{lllllllll}3766 & 3792 & 9 & 622 & 27 & 581 & 18 & 9.7 & 4.9\end{array}$

$\begin{array}{lllllllll}3080 & 3017 & 3 & 371 & 10 & 352 & 7 & 15.8 & 8.8\end{array}$

$\begin{array}{lllllllll}7389 & 7564 & 1 & 83 & 4 & 63 & 3 & 13.2 & 22.3\end{array}$

$\begin{array}{lllllllll}3034 & 2998 & 5 & 442 & 15 & 330 & 10 & 50.3 & 14.0\end{array}$

$\begin{array}{lllllllll}2903 & 2917 & 2 & 388 & 7 & 366 & 5 & 42.7 & 13.8\end{array}$

$\begin{array}{llllllllll}4645 & 4654 & 4 & 490 & 11 & 467 & 7 & 10.4 & 9.0\end{array}$

$\begin{array}{lllllllll}3443 & 3265 & 4 & 271 & 11 & 251 & 7 & 17.7 & 8.3\end{array}$

$\begin{array}{lllllllll}3624 & 3521 & 2 & 164 & 6 & 143 & 4 & 19.4 & 15.7\end{array}$

$\begin{array}{lllllllll}3350 & 3343 & 4 & 264 & 11 & 245 & 8 & 11.2 & 8.0\end{array}$

$\begin{array}{lllllllll}3548 & 3549 & 1 & 207 & 3 & 189 & 2 & 29.5 & 30.0\end{array}$

$\begin{array}{lllllllll}6076 & 6027 & 4 & 569 & 11 & 513 & 7 & 27.5 & 14.0\end{array}$

$\begin{array}{lllllllll}3710 & 3901 & 5 & 246 & 14 & 209 & 9 & 7.4 & 9.2\end{array}$

$\begin{array}{lllllllll}6233 & 6262 & 9 & 540 & 28 & 481 & 19 & 10.7 & 5.7\end{array}$

$\begin{array}{lllllllll}4968 & 4902 & 3 & 384 & 10 & 370 & 7 & 15.4 & 7.5\end{array}$

$\begin{array}{lllllllll}5910 & 5998 & 4 & 429 & 13 & 404 & 9 & 9.4 & 7.9\end{array}$

$\begin{array}{lllllllll}5025 & 5057 & 4 & 378 & 11 & 357 & 8 & 5.2 & 8.3\end{array}$

$\begin{array}{lllllllll}5367 & 5320 & 7 & 382 & 22 & 349 & 15 & 6.8 & 5.3\end{array}$
(2)

(1) 
TABLE 1. continued

\begin{tabular}{|c|c|c|c|c|c|c|c|c|c|c|c|c|}
\hline $\begin{array}{l}\text { PGC/LEDA } \\
\text { (1) }\end{array}$ & ame & $\begin{array}{l}\text { R.A. } 1950 \text { DEC. } \\
\mathrm{hms} d{ }^{\prime} \text {, " }\end{array}$ & $\begin{array}{l}\text { Vlit. } \\
\mathrm{km} / \mathrm{s} \\
(4)\end{array}$ & $\begin{array}{l}\mathrm{V} 20 \\
\mathrm{~km} / \mathrm{s} \\
(5)\end{array}$ & & $\begin{array}{l}\mathrm{W} 20 \\
\mathrm{~km} / \mathrm{s} \\
(6)\end{array}$ & & $\begin{array}{l}\text { W50 } \\
\mathrm{km} / \mathrm{s} \\
(7)\end{array}$ & & $\begin{array}{l}\mathrm{F}(\mathrm{HI}) \\
\mathrm{Jy} \cdot \mathrm{km} \\
(8)\end{array}$ & $\begin{array}{l}\mathrm{S} / \mathrm{N} \\
\mathrm{n} / \mathrm{s} \\
(9)\end{array}$ & $\begin{array}{l}\text { Note } \\
(10)\end{array}$ \\
\hline \multicolumn{13}{|c|}{$==============$} \\
\hline PGC 63007 ESO & $184-51$ & $191256.0-534724$ & 7211 & 7205 & 8 & 542 & 22 & 475 & 15 & 16.3 & 7.5 & \\
\hline PGC 63064 IC & 4843 & $191501.0-592406$ & 3972 & 3975 & 11 & 170 & 33 & 145 & 22 & 2.3 & 3.1 & \\
\hline PGC 63241 NGC & 6794 & $192439.0-390118$ & 5980 & 6019 & 9 & 409 & 26 & 365 & 18 & 8.1 & 5.2 & \\
\hline PGC 63297 ESO & $184-74$ & $192619.9-572312$ & 5059 & 5059 & 12 & 487 & 36 & 4132 & 24 & 31.9 & 4.9 & \\
\hline PGC 63509 ESO & $142-30$ & $193619.0-600948$ & 4246 & 4242 & 16 & 476 & 49 & 3993 & 33 & 11.8 & 3.7 & \\
\hline PGC 64422 ESO & $340-8$ & $201349.0-410442$ & 2852 & 2837 & 3 & 258 & 8 & 248 & 5 & 8.2 & 8.3 & \\
\hline PGC 64423 IC & 4986 & $201318.0-551124$ & 2119 & 2168 & 3 & 196 & 8 & 174 & 5 & 11.3 & 11.9 & \\
\hline PGC 64464 NGC & 6889 & $201503.0-540648$ & 2554 & 2535 & 3 & 188 & 9 & 178 & 6 & 6.6 & 7.4 & \\
\hline PGC 10329 NGC & 1073 & $024105.0+010955$ & 1209 & 1208 & & 92 & & 75 & & 72.0 & 71.0 & $(4)$ \\
\hline PGC 40761 IC & 3356 & $122421.6+115018$ & 1101 & 1103 & & 95 & & 74 & & 24.0 & 44.0 & (4) \\
\hline
\end{tabular}

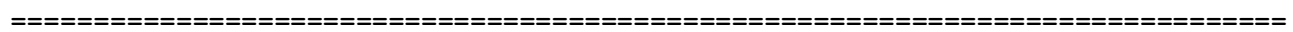

$=============$

Note: 1) Bizarre spectrum

2) W50 uncertain

3) Uncertain

4) Calibrating galaxy 

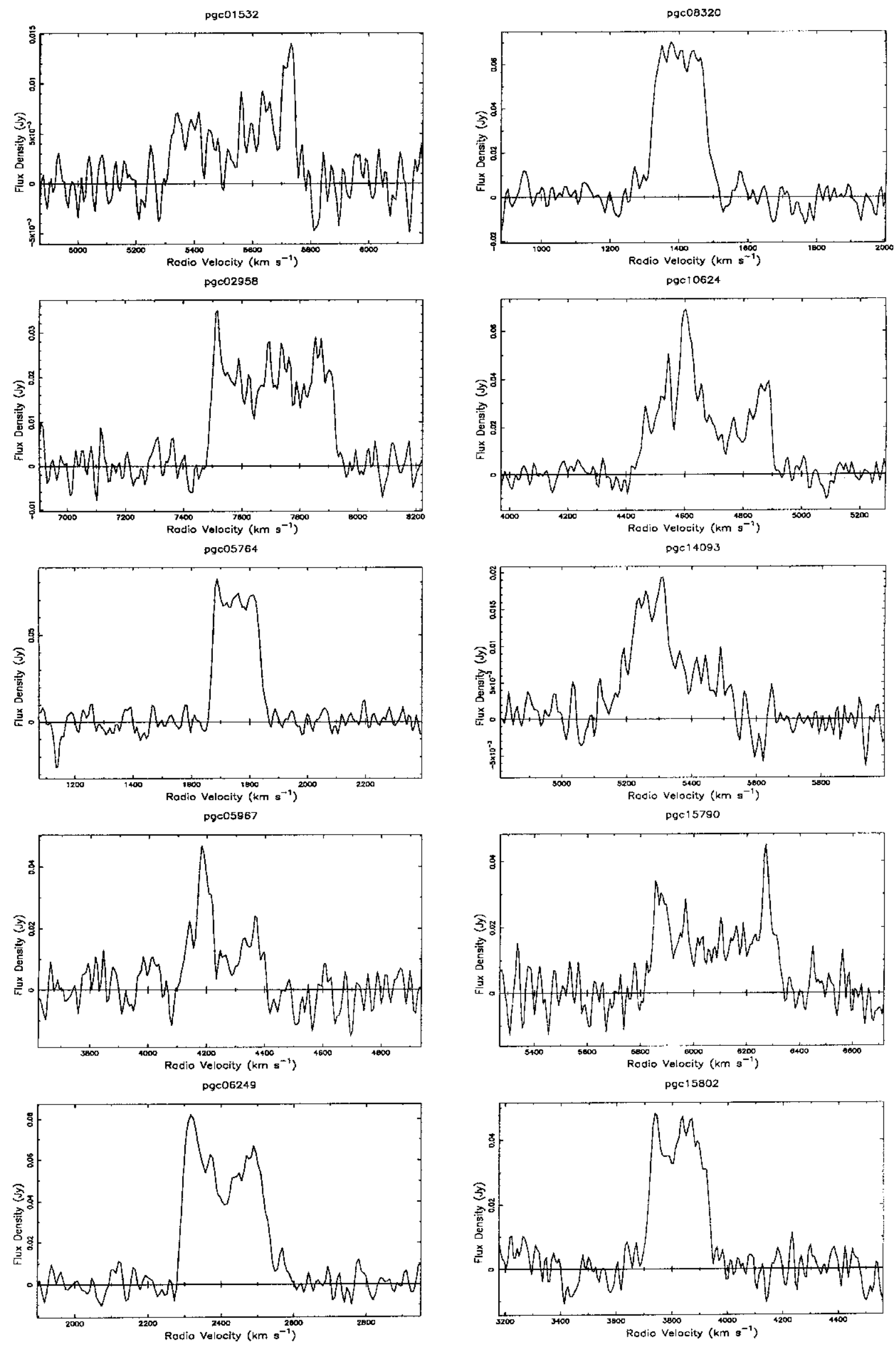

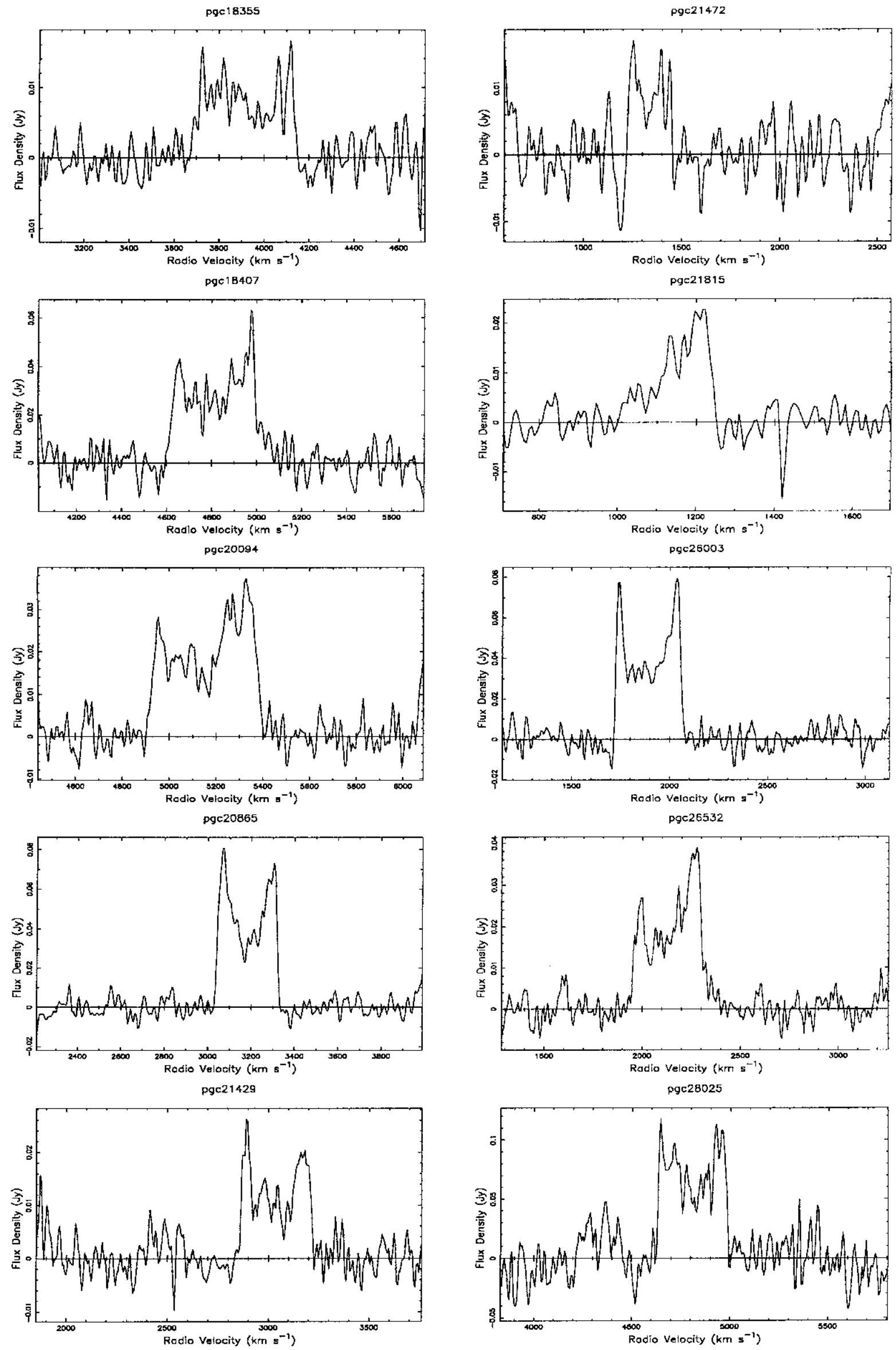

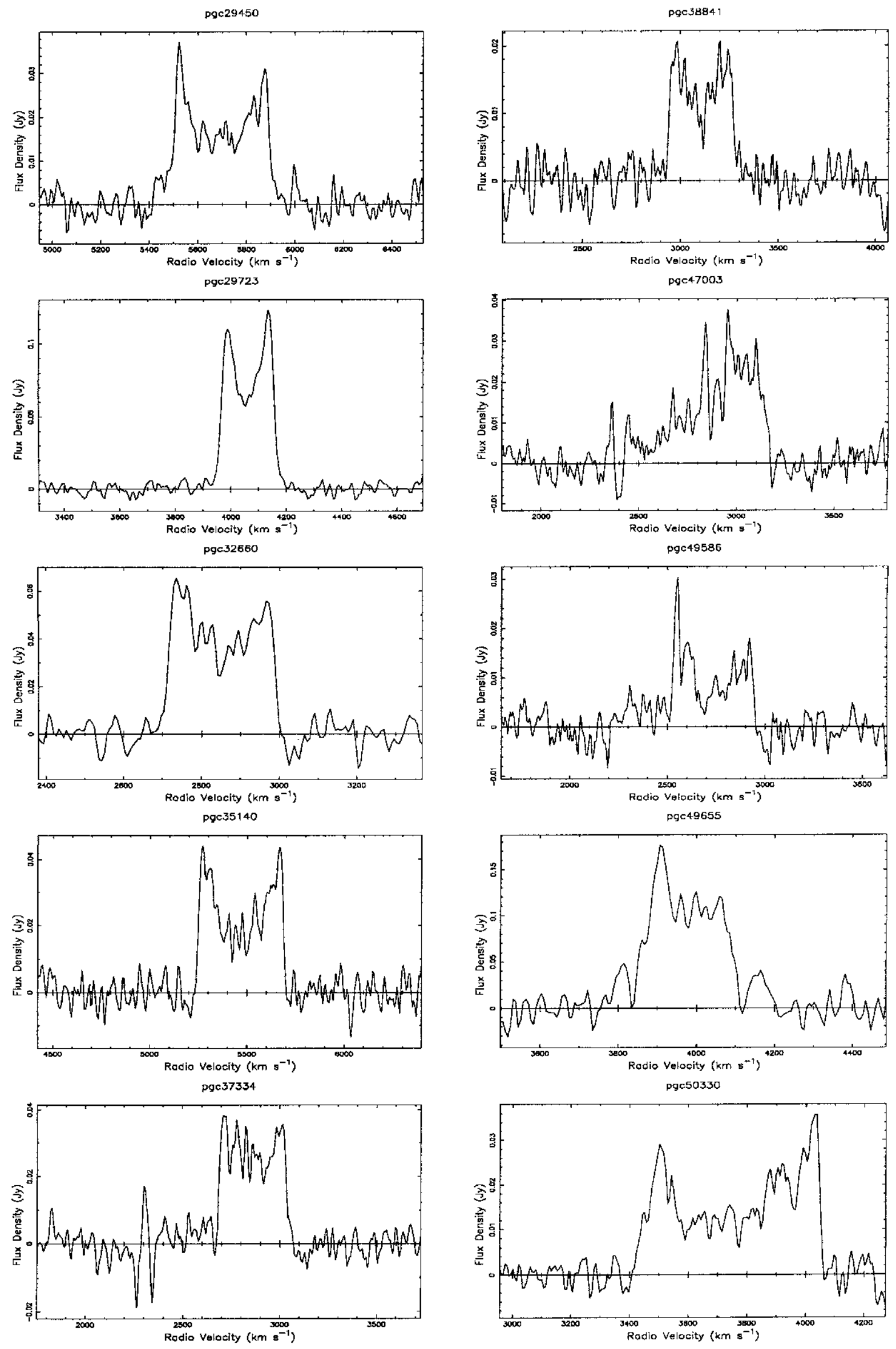

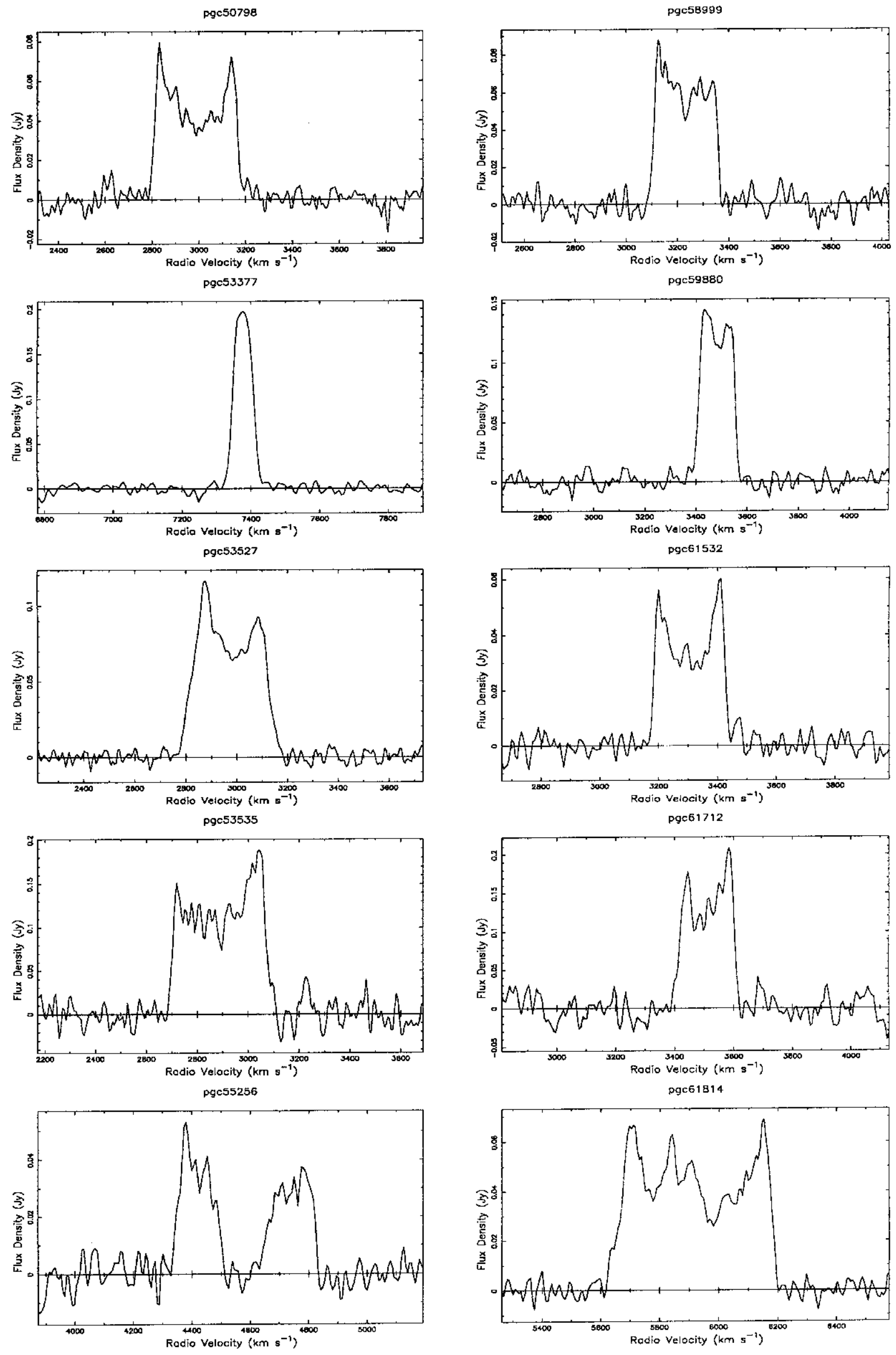

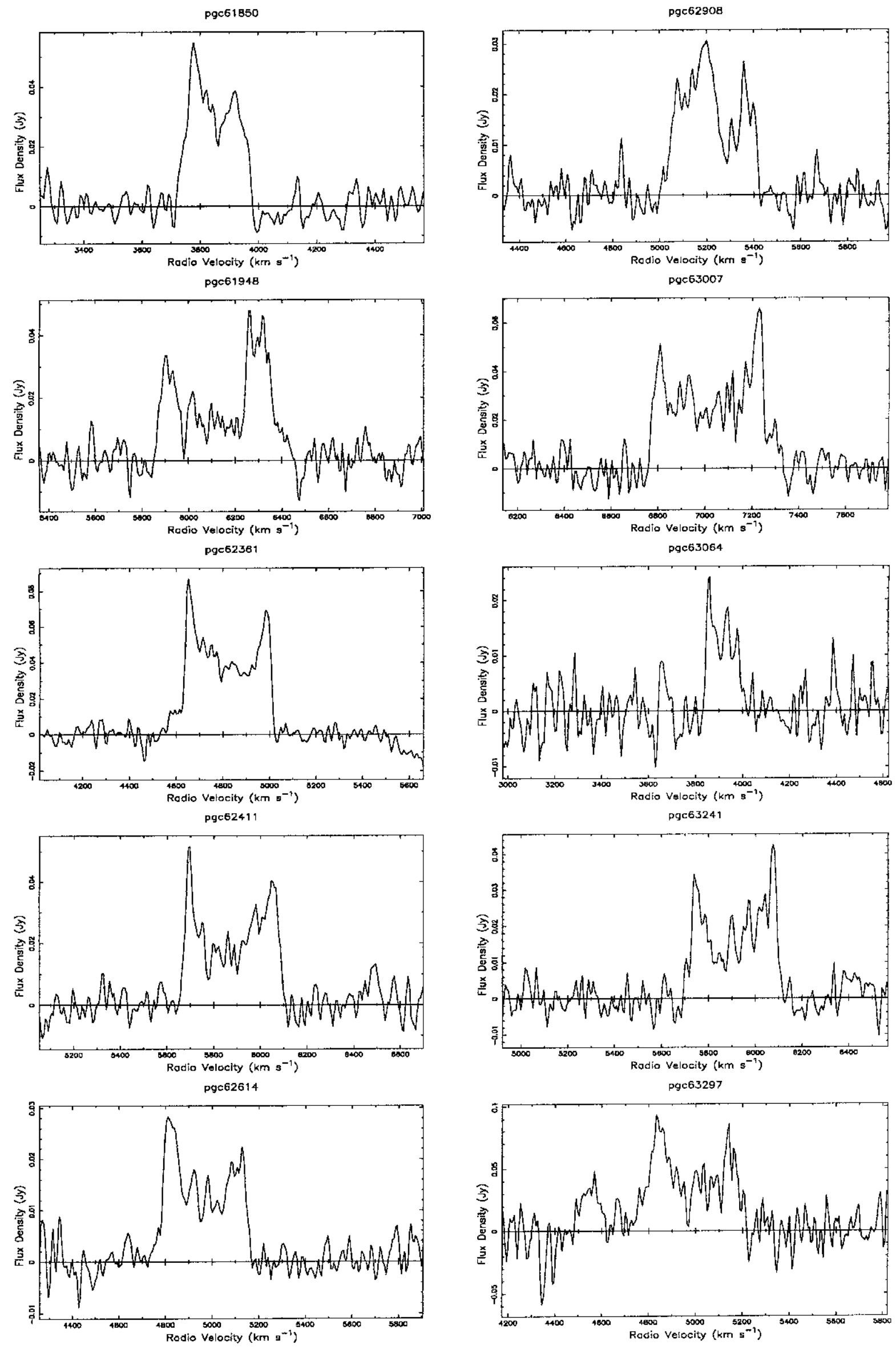

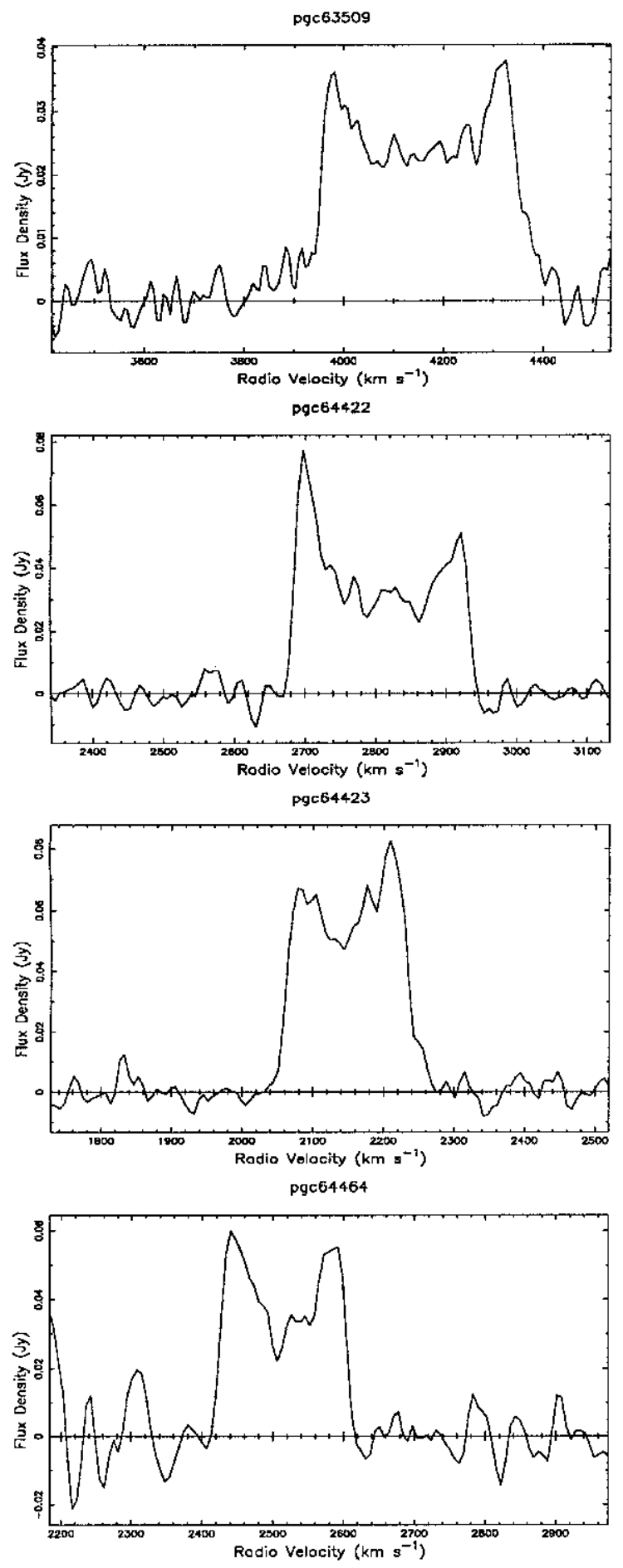